\title{
MODELING AND ANALYSIS OF AN ABSORPTION COLUMN CONNECTED TO A MICROALGAE CULTURE*
}

\author{
CARLOS MARTINEZ ${ }^{\dagger}$, ANDRÉS ÁVILA ${ }^{\ddagger}$, FRANCIS MAIRET $^{\dagger}$, LESLIE MEIER $^{\Uparrow}$, AND \\ DAVID JEISON\|
}

\begin{abstract}
We build and analyze a mathematical model of a system for biogas upgrading: an absorption column connected to a microalgae culture. The construction of the model is based on a chemical engineering approach and classical models of microalgae cultures. In our analysis, we prove the existence and uniqueness of solutions of a boundary value problem describing the absorption column. Some properties of this solution are also proved. Then, we use these results to study the long-term behavior of the mathematical model describing the coupled system. We state sufficient conditions for the existence of a globally attracting steady state characterized by the presence of microalgae. Finally, we find numerically the steady states of the system to test the efficiency of the system for purifying biogas.
\end{abstract}

Key words. biogas upgrading, absorption column, boundary value problem, global asymptotic behavior, shooting method, microalgae

AMS subject classifications. 34B60, 34A12, 92B99

DOI. $10.1137 / 18 \mathrm{M} 1225641$

1. Introduction. An absorption column $(\mathrm{AC})$ is a vertical tower that provides contact between two phases and matter transfer can happen between both phases. There are different types of ACs, such as the bubble columns, where the contact is between a rising flow of gas (as bubbles) and a downstream liquid flow. ACs have many applications [15, 27]; in particular, bubble columns are used to purify biogas [1]. Biogas is a biocombustible produced from anaerobic digestion, the composition of which is mainly carbon dioxide and methane [19]. Many of the commercial applications of biogas, such as replacement of natural gas, require increasing the calorific value removing carbon dioxide. This process is normally referred to as biogas upgrading. The removed carbon dioxide can be used as a carbon source for photosynthetic organisms such as microalgae $[6,7]$. Consequently, biogas upgrading can be done using an AC connected to a photobioreactor (PBR) with microalgae [20, 22, 32] (see Figure 2). Thus, microalgae capture carbon dioxide by photosynthetic growth. In addition to biogas upgrading, this system allows the production of microalgal biomass, which can be used as feed, food, or even biofuel $[31,35]$. Nonetheless, biogas absorbs part of the oxygen

* Received by the editors December 17, 2018; accepted for publication (in revised form) December 20, 2019; published electronically March 12, 2020.

https://doi.org/10.1137/18M1225641

Funding: This work was supported by project FONDECYT 1120488, by INRIA Chile-CIRIC, by the INRIA associated team Greencore, and by the Inria Project Lab Algae in Silico.

†Université Côte d'Azur, Inria, INRA, CNRS, UPMC Université Paris 06, Biocore Team, Sophia Antipolis, France (carlos.martinez@inria.fr).

${ }^{\ddagger}$ Centro de Excelencia de Modelación y Computación Científica, Universidad de La Frontera, Temuco, Chile, and Institut für Mathematik, Universität Kassel, Kassel, Germany (andres.avila@ ufrontera.cl, avila@mathematik.uni-kassel.de).

$\S$ IFREMER Physiology and Biotechnology of Algae Laboratory, Nantes, France (francis.mairet@ ifremer.fr).

\Department of Chemical Engineering, Universidad de La Frontera, Temuco, Chile (lmeier@ bioaqua.cl).

" Escuela de Ingeniería Bioquímica, Pontificia Universidad Católica de Valparaíso, Valparaíso, Chile (david.jeison@pucv.cl). 
produced through photosynthesis. This may induce the formation of explosive mixtures [18]. Indeed, most international standards restrict oxygen content in treated biogas. As a consequence, the system must be carefully designed and operated to maximize carbon dioxide removal while limiting oxygen transfer. Mathematical models are of great help for such a challenge.

In this work, we build and analyze a mathematical model of an AC coupled with a microalgae culture to study its long-term behavior. The model is based on a chemical engineering approach for describing the AC [21] and on classical models for describing microalgae cultures [5] featuring the main factors explaining biogas upgrading (physical phenomena) and microalgae growth (biological phenomena). Since the biogas and the liquid are injected at the bottom and at the top of the column, respectively, the $\mathrm{AC}$ is described by a boundary value problem (BVP). BVPs may present multiplicity or even nonexistence of solutions (see, for example, [4]), which may indicate a problem in model formulation. Our first result states the existence and uniqueness of solutions of this BVP. In our approach, we use the shooting method [3] and classical results of the theory of differential equations such as the comparison method [8]. The results associated to the BVP serve to analyze the coupled system and to set up a result for the persistence of microalgae. To state this result, we use the theory of monotone dynamical systems [29] and the theory of persistence [30].

As an application of our results, we determine numerically the globally attractive steady states of the coupled system to estimate the oxygen and carbon dioxide content in the treated biogas under different conditions that were considered experimentally in $[20]$.

The paper is organized as follows. In section 2 we state the mathematical model for the $\mathrm{AC}$ and we present the results related to the existence and uniqueness of solutions. In section 3, we present the mathematical model for the coupled system and we study its asymptotic behavior in section 4 . Finally, in section 5 , we test numerically the capacity of the coupled system for biogas upgrading.

2. Modeling an absorption column. Consider an AC as described by Figure 1 (see Table 1 for nomenclature), with a gas feed stream at the bottom, whose composition is carbon dioxide and methane, and with a liquid feed stream at the top

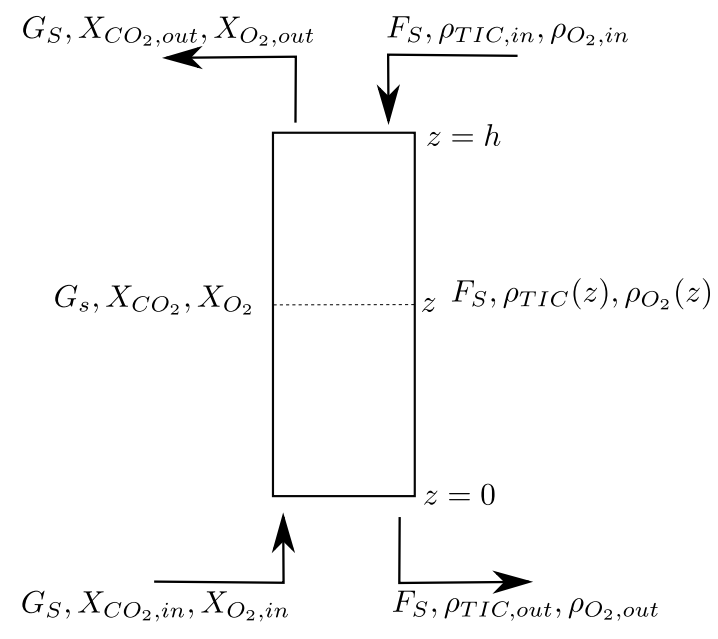

FIG. 1. Scheme of the $A C$. 
TABLE 1

Parameters and variables of the models for the $A C$ (section 2) and for the coupled system

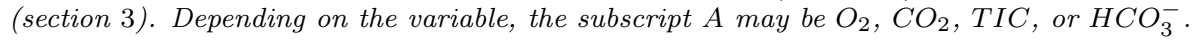

\begin{tabular}{llll}
\hline Section 2 & Section 3 & Definition & Typical units \\
\hline$X_{A}$ & - & Moles of $A$ per mol of $C H_{4}$ in the biogas & $\mathrm{mol} / \mathrm{mol}$ \\
$X_{A, \text { in }}$ & - & $X_{A}$ at the bottom of the column & $\mathrm{mol} / \mathrm{mol}$ \\
$X_{A, \text { out }}$ & - & $X_{A}$ at the top of the column & $\mathrm{mol} / \mathrm{mol}$ \\
$\rho_{A}$ & - & Concentration of $A$ in the liquid phase & $\mathrm{mol} / \mathrm{L}$ \\
$\rho_{A, \text { in }}$ & $S_{A}$ & Concentration of $A$ at the top of the column (sec. 2) or in the PBR (sec. 3$)$ & $\mathrm{mol} / \mathrm{L}$ \\
$\rho_{A, \text { out }}$ & $S_{A}^{0}$ & Concentration of $A$ at the bottom of the column (sec. 2) or as supply in the & $\mathrm{mol} / \mathrm{L}$ \\
& & PBR coming from the column (sec. 3) & $\mathrm{mol} / \mathrm{d} / \mathrm{m}^{2}$ \\
$G_{S}$ & - & Superficial flow rate of the amount of moles of $C H_{4}$ in the gas phase & $\mathrm{L} / \mathrm{d}$ \\
$F$ & $F$ & Liquid flow rate in the column or circulation flow rate in the coupled system & $\mathrm{m} / \mathrm{d}$ \\
$F_{S}$ & - & Superficial liquid flow rate in the column & $\mathrm{d}^{-1}$ \\
$\left(k_{L} a\right)_{A}$ & - & Gas-liquid transfer coefficient of $A$ & $\mathrm{~m}^{2}$ \\
$S$ & - & Cross-sectional area of the column & $\mathrm{mol} / \mathrm{L}$ \\
$\rho_{A}^{*}$ & - & Saturation concentration of $A$ & $\mathrm{~atm}$ \\
$P_{0}$ & - & Pressure inside the column & $\mathrm{d}^{-1}$ \\
- & $D$ & Dilution rate & $\mathrm{mol} / \mathrm{L}$ \\
- & $S_{A, i n}$ & PBR supply concentration of $A$ & $\mathrm{~g} / \mathrm{L}$ \\
- & $X$ & Microalgae concentration in the PBR & $\mu \mathrm{mol} \mathrm{m} \mathrm{m}^{-2} \mathrm{~s}^{-1}$ \\
- & $I_{i n}$ & Incident irradiance on the PBR & $\mathrm{m}^{3}$ \\
- & $V$ & Volume of the PBR & $\mathrm{m}$ \\
\hline
\end{tabular}

with carbon dioxide and oxygen. Experimental studies have shown negligible methane exchanges during biogas upgrading [26]. We consider the following assumption.

A 2.1. Between the gas phase and the liquid phase, there are only two possible exchanges: carbon dioxide and oxygen.

Assumption A 2.1 implies that methane concentration in the gas phase is constant along the column and the gas could have oxygen in its composition at the top of the column. Considering A 2.1 and following [34], we define $\mathrm{X}_{\mathrm{CO}_{2}}$ as the amount of moles of $\mathrm{CO}_{2}$ in the gas phase per mole of $\mathrm{CH}_{4}$,

$$
X_{\mathrm{CO}_{2}}:=\frac{x_{\mathrm{CO}_{2}}}{1-x_{\mathrm{CO}_{2}}-x_{\mathrm{O}_{2}}},
$$

where $x_{\mathrm{CO}_{2}}$ and $x_{\mathrm{O}_{2}}$ stand for the molar ratios in the gas phase of $\mathrm{CO}_{2}$ and $\mathrm{O}_{2}$, respectively. Similarly, we define $\mathrm{X}_{\mathrm{O}_{2}}$ as the amount of moles of $\mathrm{O}_{2}$ in the gas phase per mole of $\mathrm{CH}_{4}$,

$$
X_{O_{2}}:=\frac{x_{O_{2}}}{1-x_{C O_{2}}-x_{O_{2}}} .
$$

Absorption of $\mathrm{CO}_{2}$ decreases the $\mathrm{pH}$ in the liquid phase. In the context of biogas upgrading with microalgae, experimental results in [20] show that the $\mathrm{pH}$ (not controlled) in a microalgae culture remains lower than 8 . Thus, if we assume that in the liquid stream the $\mathrm{pH}$ is lower than 8 , we can neglect the presence of carbonate $\mathrm{CO}_{3}^{2-}$ [10]. This motivates the following assumption.

A 2.2. In the liquid phase, inorganic carbon is only found in the form of carbon dioxide $\left(\mathrm{CO}_{2}\right)$ and bicarbonate $\left(\mathrm{HCO}_{3}^{-}\right)$. Then, the following reversible reactions are considered:

$$
\begin{gathered}
\mathrm{CO}_{2}+\mathrm{H}_{2} \mathrm{O} \leftrightharpoons \mathrm{H}^{+}+\mathrm{HCO}_{3}^{-}, \\
\mathrm{H}_{2} \mathrm{O} \leftrightharpoons \mathrm{H}^{+}+\mathrm{OH}^{-}
\end{gathered}
$$

these are in chemical equilibrium. 
Reactions (2.3) and (2.4) are considered in equilibrium because the AC operates at a timescale of hours [20] (hydraulic retention time), while reactions (2.3) and (2.4) occur at timescales of $10 s$ and $10^{-7} \mathrm{~s}$, respectively. The $\mathrm{pH}$ varies along the column due to $\mathrm{CO}_{2}$ transfer. The following assumption is useful when considering such variations [33].

A 2.3. The liquid phase is not electrically charged (electroneutrality), i.e., the concentration of anions balanced by the number of charges must equalize the concentration of cations balanced in the same manner.

The following assumptions A 2.4-A 2.8 are taken from classical models of absortion column [21].

A 2.4. Each phase is in piston flow, which means that there is no axial mixing in the column but complete radial mixing. Complete radial mixing implies that fluid properties, including velocity, are uniform across any plane perpendicular to the flow direction.

A 2.5. The temperature and the pressure are constant along the column.

A 2.6. The operation of the column is at steady state.

A 2.7. The two-film model is applicable [17].

A 2.8. The liquid flow rate is constant throughout the column; that is, the mass transfer from one phase to the other does not affect the rate of flow for the liquid phase (the "dilute-system assumption").

The concentration of any species A in the liquid phase is denoted by $\rho_{A}(\mathrm{~mol} / L)$. An important variable in our model is the concentration of total inorganic carbon (TIC) in the liquid medium, denoted by $\rho_{\text {TIC }}$. The TIC is formed by bicarbonate $\left(\mathrm{HCO}_{3}^{-}\right)$and carbon dioxide $\left(\mathrm{CO}_{2}\right)$. Thus,

$$
\rho_{\mathrm{TIC}}=\rho_{\mathrm{CO}_{2}}+\rho_{\mathrm{HCO}_{3}^{-}} .
$$

We note that $\rho_{\text {TIC }}$ is not affected by the chemical reaction (2.3). Indeed, the production of one mol of $\mathrm{HCO}_{3}^{-}$implies the consumption of one mol of $\mathrm{CO}_{2}$, and vice versa. Thus, when doing mass balance equations, it is more convenient to work with $\rho_{T I C}$ instead of $\rho_{\mathrm{CO}_{2}}$. Let us denote by $F$ the total liquid volumetric flow rate $(L / d)$ and by $G_{S}$ the amount of moles of $\mathrm{CH}_{4}$ in the gas phase per day per cross-sectional area of the tower. The continuity equation in the liquid phase and the overall material balance along the column for each species (TIC and $\mathrm{O}_{2}$ ) give the following system of differential equations (for more details see [34]):

$$
\begin{aligned}
& \frac{F}{S} \frac{d \rho_{T I C}}{d z}=G s \frac{d X_{C O_{2}}}{d z} \\
& \frac{F}{S} \frac{d \rho_{T I C}}{d z}=\left(k_{L} a\right)_{C O_{2}}\left(\rho_{C O_{2}}-\rho_{C O_{2}}^{*}\right) \\
& \frac{F}{S} \frac{d \rho_{O_{2}}}{d z}=G s \frac{d X_{O_{2}}}{d z} \\
& \frac{F}{S} \frac{d \rho_{O_{2}}}{d z}=\left(k_{L} a\right)_{O_{2}}\left(\rho_{O_{2}}-\rho_{O_{2}}^{*}\right)
\end{aligned}
$$

where $\left(k_{L} a\right)_{C_{2}}$ and $\left(k_{L} a\right)_{O_{2}}$ are gas-liquid transfer coefficients depending on the hydrodynamic conditions and the diffusivities. $S$ is the cross-sectional area of the 
column. $\rho_{\mathrm{CO}_{2}}^{*}$ and $\rho_{\mathrm{O}_{2}}^{*}$ are the saturation concentrations that can be obtained from Henry's law and the definition of the variables $\mathrm{X}_{\mathrm{CO}_{2}}, \mathrm{X}_{\mathrm{O}_{2}}$, i.e.,

$$
\begin{gathered}
\rho_{C O_{2}}^{*}=H_{C_{2}} P_{0} x_{C_{2}}=H_{C_{2}} P_{0} \frac{X_{C O_{2}}}{1+X_{C_{2}}+X_{O_{2}}}, \\
\rho_{O_{2}}^{*}=H_{O_{2}} P_{0} x_{O_{2}}=H_{O_{2}} P_{0} \frac{X_{O_{2}}}{1+X_{C_{2}}+X_{O_{2}}},
\end{gathered}
$$

where $\mathrm{H}_{\mathrm{CO}_{2}}$ and $\mathrm{H}_{\mathrm{O}_{2}}$ are the Henry's constants and $P_{0}$ is the pressure that, from A 2.5, is constant along the column. In the following we will write $F_{S}$ instead of $F / S$.

System (2.6) has four differential equations and five state variables. Based on assumptions A 2.2 and A 2.3, we can write $\rho_{\mathrm{CO}_{2}}$ in terms of $\rho_{T I C}$. Indeed, from assumption A 2.2, we get the equations

$$
K_{C}=\frac{\rho_{H^{+}} \rho_{\mathrm{HCO}_{3}^{-}}}{\rho_{C O_{2}}} \text { and } K_{W}=\rho_{H^{+}} \rho_{O H^{-}},
$$

where $K_{C}$ and $K_{W}$ are the acid dissociation constants. From assumption A 2.3, we have that

$$
\rho_{H^{+}}+Z=\rho_{O H^{-}}+\rho_{\mathrm{HCO}_{3}^{-}},
$$

where $Z$ is the inert charge imbalance, defined as the sum of cation concentrations minus anion concentrations not affected by the process, multiplied by their respective valency. Apart from $Z$, all the concentrations (five variables) involved in (2.5), (2.9), and (2.10) are not constant along the column. We must write $\rho_{\mathrm{CO}_{2}}$ only in terms of $\rho_{\text {TIC }}$ and constant terms. By (2.5), (2.9), and (2.10), we get

$$
\frac{K_{C} \rho_{C O_{2}}}{\left(\rho_{T I C}-\rho_{C O_{2}}\right)}+Z=\frac{K_{W}\left(\rho_{T I C}-\rho_{C O_{2}}\right)}{K_{C} \rho_{C O_{2}}}+\rho_{T I C}-\rho_{C O_{2}}
$$

The following proposition shows that for any value of $\rho_{T I C}$, there is a unique value of $\rho_{\mathrm{CO}_{2}}$ satisfying (2.11).

Proposition 2.9. Assume that (2.11) holds. Then, there is a unique continuous function $\phi: \mathbb{R}_{+} \longrightarrow \mathbb{R}_{+}$such that $\rho_{C O_{2}}=\phi\left(\rho_{T I C}\right)$. The function $\phi$ satisfies

$$
0<\phi^{\prime}\left(\rho_{T I C}\right) \leq 1 \text { and } \phi\left(\rho_{T I C}\right) \leq \rho_{T I C} \text { for all } \rho_{T I C} \geq 0,
$$

and $\lim _{\rho_{T I C} \rightarrow \infty} \phi\left(\rho_{T I C}\right)=\infty$.

Proof. From (2.11), we can easily solve $\rho_{T I C}$ as a function of $\rho_{C O_{2}}$, that is, $\rho_{\text {TIC }}=\varphi\left(\rho_{\mathrm{CO}_{2}}\right)$, where

$$
\varphi\left(\rho_{\mathrm{CO}_{2}}\right)=\rho_{\mathrm{CO}_{2}}\left(1+\frac{Z+\sqrt{Z^{2}+4 K_{W}+4 K_{C} \rho_{C O_{2}}}}{2\left(b+\rho_{C O_{2}}\right)}\right)
$$

with $b=K_{W} / K_{C}$. We claim that $\varphi:[0, \infty) \longrightarrow[0, \infty)$ is a homeomorphism. Indeed, we compute

$$
\begin{aligned}
& \varphi^{\prime}\left(\rho_{\mathrm{CO}_{2}}\right) \\
& \quad=1+\frac{b Z\left(Z+\sqrt{Z^{2}+4 K_{C}\left(\rho_{C O_{2}}+b\right)}\right)+2 K C\left(b+\rho_{C O_{2}}\right)\left(2 b+\rho_{C O_{2}}\right)}{2\left(b+\rho_{C O_{2}}\right)^{2} \sqrt{Z^{2}+4 K_{C}\left(\rho_{C O_{2}}+b\right)}} \geq 1 .
\end{aligned}
$$

Copyright (c) by SIAM. Unauthorized reproduction of this article is prohibited. 
Then, $\varphi$ is strictly increasing and hence injective. Now, we note that

$$
\varphi(0)=0 \text { and } \lim _{\rho_{\mathrm{CO}_{2} \rightarrow \infty}} \varphi\left(\rho_{\mathrm{CO}_{2}}\right)=\infty .
$$

Thus $\varphi([0, \infty))=[0, \infty)$ and $\varphi$ is surjective. Finally, since $\varphi$ is continuous, we conclude the claim. We will denote the inverse of $\varphi$ by $\phi$. Thus, $\rho_{C_{2}}=\phi\left(\rho_{T I C}\right)$. Since $\varphi\left(\rho_{\mathrm{CO}_{2}}\right) \geq \rho_{C \mathrm{O}_{2}}$, it follows that $\phi\left(\rho_{\text {TIC }}\right) \leq \rho_{T I C}$. It remains to prove the first inequality of (2.12). By the inverse function theorem [24] and (2.14), we obtain

$$
0<\phi^{\prime}\left(\rho_{T I C}\right)=\frac{1}{\varphi^{\prime}\left(\phi\left(\rho_{T I C}\right)\right)} \leq 1
$$

Remark 2.10. Note that $\phi$ depends on $Z$. In some cases, it is convenient to specify the dependence of $\phi$ on $Z$ writing $\phi\left(\rho_{\text {TIC }} ; Z\right)$ instead of $\phi\left(\rho_{T I C}\right)$.

Proposition 2.9 shows that $\phi$ is strictly increasing and Lipschitz and that $\rho_{T I C}$ is not lower than $\rho_{\mathrm{CO}_{2}}$. Natural boundary conditions for system (2.6) are (see Figure 1)

$$
\begin{gathered}
\rho_{T I C}(h)=\rho_{T I C, i n}, \quad X_{C O_{2}}(0)=X_{C O_{2}, i n}, \\
\rho_{O_{2}}(h)=\rho_{O_{2}, i n}, \quad X_{O_{2}}(0)=X_{O_{2}, i n} .
\end{gathered}
$$

Thus, we have a BVP formed by system (2.6) and the boundary conditions (2.16)(2.17).

In the context of biogas purification, the injected biogas in the column usually has a neglected concentration of oxygen; therefore we assume that $X_{O_{2}, \text { in }}=0$. Since a small amount of oxygen is transferred from the liquid to the gas, we neglect $X_{\mathrm{O}_{2}}$ in the expression for $\rho_{T I C}^{*}$, that is,

$$
\rho_{\mathrm{CO} 2}^{*}=\mathrm{H}_{\mathrm{CO}_{2}} P_{0} \frac{X_{\mathrm{CO}_{2}}}{1+X_{\mathrm{CO}_{2}}} .
$$

In this way, we obtain the following two-dimensional ODE for the TIC, which is decoupled from the equations for the oxygen:

$$
\begin{aligned}
\frac{d X_{C O_{2}}}{d z} & =\frac{\left(k_{L} a\right)_{C O_{2}}}{G_{s}}\left(\phi\left(\rho_{T I C}\right)-H_{C O_{2}} P_{0} \frac{X_{C O_{2}}}{1+X_{C O_{2}}}\right) \\
\frac{d \rho_{T I C}}{d z} & =\frac{\left(k_{L} a\right)_{C O_{2}}}{F_{s}}\left(\phi\left(\rho_{T I C}\right)-H_{C_{2}} P_{0} \frac{X_{C O_{2}}}{1+X_{C O_{2}}}\right) .
\end{aligned}
$$

Our first theorem states the existence and uniqueness of solutions for the BVP (2.16)(2.18) and gives some properties of the solutions. The proof is given at the end of this section.

THEOREM 2.11. BVP (2.16)-(2.18) has a unique nonegative solution $\left(\bar{X}_{\mathrm{CO}_{2}}\right.$, $\left.\bar{\rho}_{T I C}\right)$ satisfying $\bar{X}_{\mathrm{CO}_{2}}(0)=X_{\mathrm{CO}_{2}, \text { in }}$ and $\bar{\rho}_{T I C}(h)=\rho_{T I C, \text { in }}$. Given $\rho_{C \mathrm{O}_{2}, \text { in }}^{*}=H_{\mathrm{CO}_{2}}$ $P_{0} \frac{X_{C O_{2}, \text { in }}}{1+X_{C_{2}, \text { in }}}$, we have

(a) if $\phi\left(\rho_{T I C, \text { in }}\right)<\rho_{C O_{2}, \text { in }}^{*}$, then $\rho_{T I C, \text { out }}>\rho_{T I C, \text { in }}$ and $X_{C O_{2}, \text { out }}<X_{C O_{2}, \text { in }}$,

(b) if $\phi\left(\rho_{T I C, \text { in }}\right)=\rho_{C O_{2}, \text { in }}^{*}$, then $\rho_{T I C, \text { out }}=\rho_{T I C, \text { in }}$ and $X_{C O_{2}, \text { out }}=X_{C O_{2}, \text { in }}$,

(c) if $\phi\left(\rho_{T I C, \text { in }}\right)>\rho_{C O_{2}, \text { in }}^{*}$, then $\rho_{T I C, \text { out }}<\rho_{T I C, \text { in }}$ and $X_{C O_{2}, \text { out }}>X_{C O_{2}, \text { in }}$.

Copyright $@$ by SIAM. Unauthorized reproduction of this article is prohibited. 
Moreover,

$$
0 \leq \frac{\partial \rho_{T I C}(0)}{\partial \rho_{T I C, \text { in }}} \leq 1
$$

Theorem 2.11 not only states the existence of solutions for the BVP (2.16)(2.18) but determines if any concentration increases, decreases, or stays steady passing through the column.

Now, let $\left(\bar{X}_{C O_{2}}, \bar{\rho}_{T I C}\right)$ be the unique nonnegative solution of (2.16)-(2.18). Then, we have the following equation for the oxygen:

$$
\begin{aligned}
\frac{d X_{O_{2}}}{d z} & =\frac{\left(k_{L} a\right)_{O_{2}}}{G_{s}}\left(\rho_{O_{2}}-H_{O_{2}} P_{0} \frac{X_{O_{2}}}{1+X_{O_{2}}+\bar{X}_{C O_{2}}}\right) \\
\frac{d \rho_{O_{2}}}{d z} & =\frac{\left(k_{L} a\right)_{O_{2}}}{F_{s}}\left(\rho_{O_{2}}-H_{O_{2}} P_{0} \frac{X_{O_{2}}}{1+X_{O_{2}}+\bar{X}_{C O_{2}}}\right) .
\end{aligned}
$$

The following theorem is analogous to Theorem 2.11, but for BVP (2.17)-(2.20).

THEOREM 2.12. BVP $(2.17)-(2.20)$ has a unique nonegative solution $\left(\bar{X}_{\mathrm{O}_{2}}, \bar{\rho}_{\mathrm{O}_{2}}\right)$ satisfying $\bar{X}_{\mathrm{O}_{2}}(0)=0$ and $\bar{\rho}_{\mathrm{O}_{2}}(0)=\rho_{\mathrm{O}_{2}, \text { in }}$. We have that

(a) if $\rho_{O_{2}, \text { in }}=0$, then $\rho_{O_{2}, \text { out }}=0$ and $X_{O_{2}, \text { out }}=0$,

(b) if $\rho_{O_{2}, \text { in }}>0$, then $\rho_{O_{2}, \text { out }} \leq \rho_{O_{2}, \text { in }}$ and $X_{O_{2}, \text { out }} \geq 0$.

Moreover,

$$
0 \leq \frac{\partial \rho_{O_{2}}(0)}{\partial \rho_{O_{2}, i n}} \leq 1
$$

From Theorem 2.12 (case (b)), a part of oxygen in the liquid phase may be transferred to the gas phase. For the inorganic carbon, Theorem 2.11 shows that depending on the input concentrations, the amount of $\mathrm{CO}_{2}$ in the biogas could increase (case $(\mathrm{c})$ ), which is not desirable for biogas upgrading.

Equations (2.19) and (2.21) are relevant when coupling the model of the $\mathrm{AC}$ with that of a microalgae culture where $\rho_{T I C, \text { in }}$ corresponds to the concentration of TIC moving from the culture to the column, and $\rho_{\text {TIC }}(0)$ corresponds to an input concentration in the culture coming from the column. Thus, the term $R=F\left(\rho_{T I C}(0)-\rho_{T I C, \text { in }}\right)$ can be seen as a "circulation term," and it must be included in the mass balances of the microalgae culture. From (2.19), we obtain that

$$
\frac{\partial R}{\partial \rho_{T I C, \text { in }}} \leq 0
$$

This property is necessary in section 3 for studying the number of steady states of the coupled system. We can also see that

$$
R \leq \beta h
$$

with $\beta=L H_{C_{2}} P_{0} \frac{\left(k_{L} a\right)_{C_{2}}}{F_{s}}>0$. Indeed, from the second equation in (2.18), we have that $\frac{d \rho_{T I C}}{d z} \geq-\beta / L$. Integrating both sides of this inequality on the interval $[0, h]$ gives $(2.23)$.

We end this section with the proof of Theorems 2.11 and 2.12 .

Copyright $@$ by SIAM. Unauthorized reproduction of this article is prohibited. 
Proof of Theorem 2.11. We will denote $X_{\mathrm{CO}_{2}}$ and $S_{T I C}$ by $x$ and $y$, respectively. The BVP (2.16)-(2.18) can be put in the following form:

$$
\begin{aligned}
\frac{d x}{d z} & =\alpha[g(y)-f(z, x)], \\
\frac{d y}{d z} & =g(y)-f(z, x), \\
x(0) & =x_{0}, y(h)=y_{1}, h>0,
\end{aligned}
$$

with $\alpha>0, x_{0}, y_{1} \geq 0$ appropriate constants, $f:[0, h] \times \mathbb{R}_{+} \longrightarrow \mathbb{R}$ and $g: \mathbb{R}_{+} \longrightarrow \mathbb{R}$ defined by

$$
g(y)=k_{1} \phi(y) \text { and } f(z, x)=k_{2} \frac{x}{1+x},
$$

with $\phi$ defined in Proposition 2.9, and $k_{1}, k_{2}$, again, two appropriate positive constants. Then, we first have to prove the following: For any $x_{0}, y_{1} \geq 0$, the BVP $(2.24)$ has a unique solution $(\bar{x}, \bar{y})$. Moreover

(a) if $g\left(y_{1}\right)=f\left(x_{0}\right)$, then $\bar{x}(h)=x_{0}$ and $\bar{y}(0)=y_{1}$,

(b) if $g\left(y_{1}\right)<f\left(x_{0}\right)$, then $\bar{x}(h)>x_{0}$ and $\bar{y}(0)<y_{1}$,

(c) if $g\left(y_{1}\right)>f\left(x_{0}\right)$, then $\bar{x}(h)<x_{0}$ and $\bar{y}(h)>y_{1}$.

For this purpose, we define $g$ and $f$ for negative values of $x$ and $y$ in the following way: $g(y):=-g(-y)$ and $f(z, x):=-f(z,-x)$ for all $z \in[0, h]$ and $x, y \in[0,-\infty)$. Now, replacing $\frac{d y}{d z}$ in the first equality in (2.24) and integrating, we obtain

$$
x(z)=x_{0}+\alpha(y(z)-y(0)) .
$$

Replacing (2.26) in (2.24), we obtain the one-dimensional problem

$$
\left\{\begin{aligned}
\frac{d y}{d z} & =G(z, y, y(0)), \\
y(h) & =y_{1}
\end{aligned}\right.
$$

where $G:[0, h] \times \mathbb{R}^{2} \longrightarrow \mathbb{R}$ is given by

$$
G(z, y, \gamma)=g(y)-f\left(z, x_{0}+\alpha(y-\gamma)\right) .
$$

We have that $g(0)=0, \lim _{y \rightarrow \infty} g(y)=\infty$, and $g$ is strictly increasing. This implies the existence of $\gamma^{*} \geq 0$ such that $g\left(\gamma^{*}\right)=k_{2} \frac{x_{0}}{1+x_{0}}$ or equivalently $G\left(z, \gamma^{*}, \gamma^{*}\right)=0$. Thus, we can easily verify that $G$ satisfies the hypotheses of Lemma A.1 in the appendix; then the problem (2.27) admits a unique solution $\bar{y}$. Let us define $\bar{x}(z):=x_{0}+$ $\alpha(\bar{y}(z)-\bar{y}(0))$. If $\bar{x}$ and $\bar{y}$ are nonnegative, then $(\bar{x}, \bar{y})$ form a solution of (2.24). Let us assume that $y_{1}<\gamma^{*}$. Since $G$ does not depend on $z$, by Lemma A.1, $\bar{y}$ is strictly decreasing. Since $\bar{y}(h)=y_{1} \geq 0$, we conclude that $\bar{y}$ cannot be negative. If $y_{1} \geq \gamma^{*}$, again from Lemma A.1, we have that $\bar{y}$ is not smaller than $\gamma^{*} \geq 0$ for any $z$. Thus, $\bar{y}$ is nonnegative. With respect to $\bar{x}$, if there is a $z^{*} \in[0, h]$ such that $\bar{x}\left(z^{*}\right)=0$, from (2.24), it follows that $d \bar{x}\left(z^{*}\right) / d z=\alpha \hat{g}\left(\bar{y}\left(z^{*}\right)\right) \geq 0$. Thus, $\bar{x}$ cannot be negative, and hence $(\bar{x}, \bar{y})$ is a solution of the BVP (2.24). The uniqueness of this solution follows from the uniqueness of $\bar{y}$. Now, we prove the properties of the solution: (a) If $g\left(y_{1}\right)=f\left(x_{0}\right)$, then $y_{1}=\gamma^{*}$. From Lemma A.1, $\bar{y}(z)=y_{1}$. (b) If $g\left(y_{1}\right)<f\left(x_{0}\right)$, then $y_{1}<\gamma^{*}$. From Lemma A.1, it follows that $\bar{y}(z)$ and $\bar{x}(z)$ (see, the definition of $\bar{x}$ ) are strictly increasing, which implies (b). The proof of (c) is similar to that of (b).

Copyright (c) by SIAM. Unauthorized reproduction of this article is prohibited. 
Now, it remains to prove that $\bar{y}(0)$ is differentiable by $y_{1}$ and

$$
0 \leq \frac{\partial \bar{y}(0)}{\partial y_{1}} \leq 1
$$

Indeed, since $f, g \in C^{1}[0, \infty)$ and

$$
\frac{\partial}{\partial y} G(z, y, \gamma)+\frac{\partial}{\partial \gamma} G(z, y, \gamma)=g^{\prime}(y)>0
$$

from Lemma A.2 we conclude that $\bar{y}(0)$ is differentiable by $y_{1}$ and $0 \leq \partial \bar{y}(0) /$ $\partial y_{1} \leq 1$.

Proof of Theorem 2.12. The proof follows the same idea of that of Theorem 2.11. In (2.24), take $x_{0}=0$ and $f$ and $g$ as

$$
g(y)=k_{1} y \text { and } f(z, x)=k_{2} \frac{x}{1+x+\beta(z)},
$$

where $\beta:[0, h] \longrightarrow[0, \infty)$ is an appropriate continuous function and $k_{1}, k_{2}$ two positive appropriate constants.

3. Modeling a column absorption connected to a microalgae culture. Consider the system described by Figure 2 (see Tables 1 and 2 for nomenclature). The left side corresponds to an AC and the right side to a PBR with a microalgae culture. In this system, the flow of liquid $(F)$ in the AC comes from the PBR and then returns to the $\mathrm{PBR}$, so that microalgae use the absorbed carbon dioxide from biogas as a substrate and release oxygen to the environment. Given that column dynamics is faster than microalgae growth, we assume that the column operates at steady state.

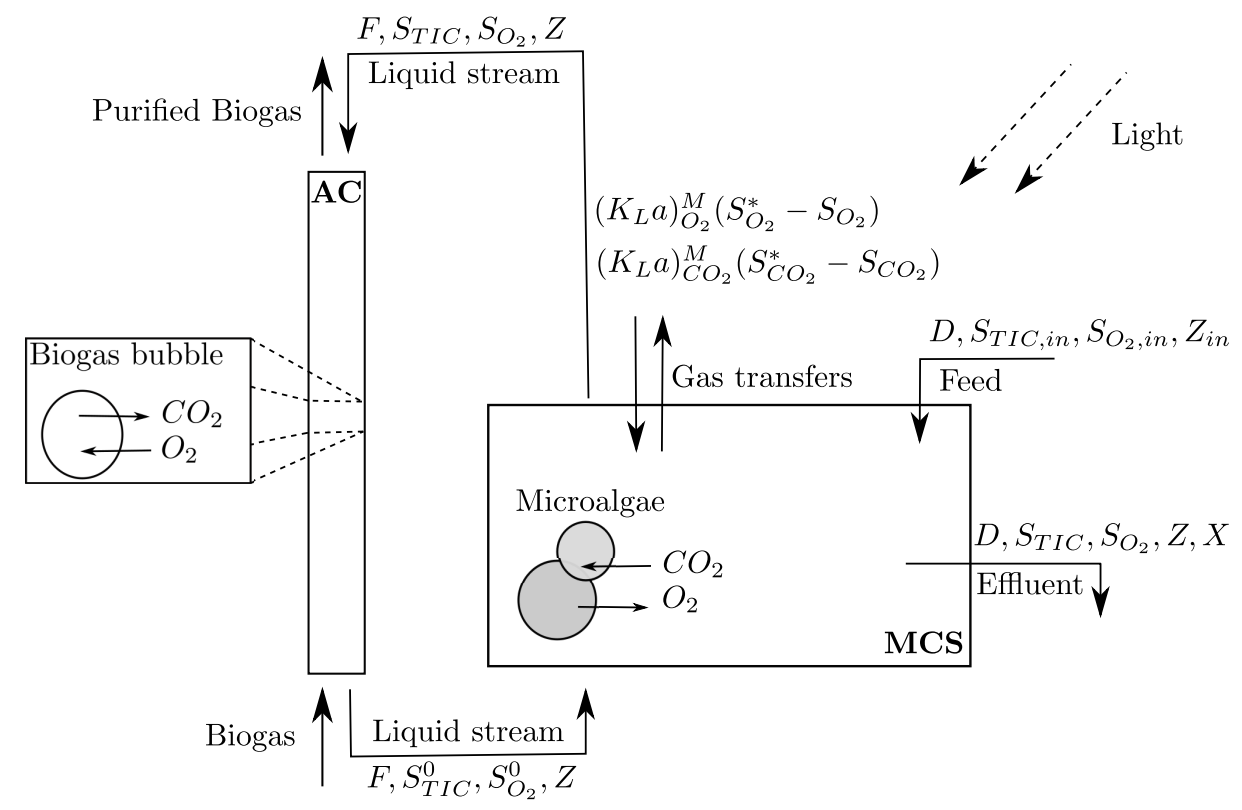

FIG. 2. Scheme of the $A C$ connected to a microalgae culture showing transfers in the $A C$ and the conversion of carbon dioxide into oxygen in the PBR.

Copyright $\odot$ by SIAM. Unauthorized reproduction of this article is prohibited. 
TABLE 2

Parameters for numerical simulation of (3.4)-(3.5). $Y_{\mathrm{CO}_{2}}$ and $\mathrm{Y}_{\mathrm{O}_{2}}$ are theoretical values assuming that one mole of $\mathrm{CO}_{2}$ gives one mole of biomass (with $0.5 \mathrm{gC} / \mathrm{g}$ biomass) and one mole of $\mathrm{O}_{2}$.

\begin{tabular}{|c|c|c|c|c|}
\hline Parameter & Definition & Value & Unit & Remark \\
\hline$\mu_{\max }$ & Maximal growth rate of microalgae & 1.68 & $d^{-1}$ & [13] C. vulgaris \\
\hline$K_{I}$ & Light half-saturation & 107 & $\mu \mathrm{mol} \mathrm{m}{ }^{-2} \mathrm{~s}^{-1}$ & [13] C. vulgaris \\
\hline$k$ & Microalgae specific attenuation coefficient & 0.1245 & $m^{2} g^{-1}$ & [12] C. vulgaris \\
\hline$K_{b g}$ & Background turbidity & 7.2 & $m^{-1}$ & {$[13]$} \\
\hline $\mathrm{K}_{\mathrm{CO}_{2}}$ & $\mathrm{CO}_{2}$ half-saturation & 0.3 & $\mu m o l L^{-1}$ & [23] C. vulgaris \\
\hline $\mathrm{Y}_{\mathrm{CO}_{2}}$ & Carbon dioxide yield coefficient & 24 & $\mathrm{~g} / \mathrm{mol} \mathrm{CO} 2$ & See caption \\
\hline$Y_{O_{2}}$ & Oxygen yield coefficient & 24 & $\mathrm{~g} / \mathrm{mol} \mathrm{O}_{2}$ & See caption \\
\hline$\left(K_{L} a\right)_{C O_{2}}^{P}$ & $\mathrm{CO}_{2}$ volumetric mass transfer coefficient (PBR-environment) & 96 & $d^{-1}$ & {$[20]$} \\
\hline$\left(K_{L} a\right)_{O_{2}}^{P}$ & $\mathrm{O}_{2}$ volumetric mass transfer coefficient (PBR-environment) & 86.4 & $d^{-1}$ & {$[20]$} \\
\hline $\mathrm{S}_{\mathrm{CO} 2}^{*}$ & Concentration of saturation in dissolved $\mathrm{CO}_{2}$ & 7.36 & $\mu \mathrm{mol} L^{-1}$ & {$[9]$} \\
\hline$S_{Q 2}^{*}$ & Concentration of saturation in dissolved $\mathrm{O}_{2}$ & $2.8199 \times 10^{-4}$ & $m o l L^{-1}$ & {$[9]$} \\
\hline $\mathrm{D}_{\mathrm{CO} 2}$ & Diffusivity of $\mathrm{CO}_{2}$ in the liquid & $2.41 \times 10^{-9}$ & $m^{2} s^{-1}$ & {$[16]$} \\
\hline $\mathrm{D}_{\mathrm{O} 2}$ & Diffusivity of $\mathrm{O}_{2}$ in the liquid & $2.0 \times 10^{-9}$ & $m^{2} s^{-1}$ & {$[16]$} \\
\hline$-\log _{10}\left(K_{C}\right)$ & Dissociation constant for $\mathrm{CO}_{2}$ & 6.352 & & {$[11]$} \\
\hline$-\log _{10}\left(K_{W}\right)$ & Dissociation constant of $\mathrm{H}_{2} \mathrm{O}$ & 14 & & {$[11]$} \\
\hline $\mathrm{H}_{\mathrm{CO}}$ & Henry constant for $\mathrm{CO}_{2}$ & 0.03344 & $m o l L^{-1} a_{t m}^{-1}$ & {$[25]$} \\
\hline $\mathrm{H}_{\mathrm{O}_{2}}$ & Henry constant for $\mathrm{CO}_{2}$ & 0.0012 & $\mathrm{~mol} L^{-1} \mathrm{~atm}^{-1}$ & {$[25]$} \\
\hline
\end{tabular}

Thus, we can describe the AC with the BVP presented in section 2 (see (2.18) and $(2.20))$.

The PBR is operating in continuous mode and constant stirring is keeping the concentrations homogeneous in the medium. The PBR is illuminated with an irradiance $I_{i n}$. In real applications, the PBR is illuminated with sunlight [26]. This may lead to a very complicated analysis of the model. Thus, we consider constant irradiance as in the lab-scale system in [20]. Light penetrating the culture decreases as it passes through the culture due to absorption and scattering by algal cells and other substances. We assume that light intensity decays exponentially with depth according to the Lambert-Beer law, i.e., at a distance $z$ from the illuminated surface, the light intensity is

$$
I(z, X)=I_{i n} e^{-z\left(k X+K_{b g}\right)}, z \in[0, L],
$$

where $X$ is the microalgae concentration, $k$ is the specific light attenuation coefficient of microalgae, $K_{b q}$ is the background turbidity, and $L$ is the depth of the PBR. We assume that microalgae growth depends on the availability of light and $\mathrm{CO}_{2}$. Thus, by using the Monod model for both limitations, we can describe the growth of microalgae at a distance $z$ from the illuminated surface by

$$
\mu_{z}(\cdot)=\mu_{\max } \frac{S_{\mathrm{CO}_{2}}}{K_{\mathrm{CO}_{2}}+S_{\mathrm{CO}_{2}}} \frac{I(z, X)}{I(z, X)+K_{I}}
$$

with $\mu_{\max }$ the maximal specific growth rate, $\mathrm{S}_{\mathrm{CO}_{2}}$ the $\mathrm{CO}_{2}$ concentration in the $\mathrm{PBR}$, and $K_{I}$ and $\mathrm{K}_{\mathrm{CO}_{2}}$ half saturation constants. Following [13], we define $\mu$ as the average of the local growth rates along the depth, i.e., $\mu(\cdot)=\frac{1}{L} \int_{0}^{L} \mu_{z}(\cdot) d z$. A simple computation gives

$$
\mu(\cdot)=\frac{\mu_{\max }}{\left(k X+K_{b g}\right) L} \ln \left(\frac{K_{I}+I_{\text {in }}}{K_{I}+I_{\text {out }}(X)}\right) \frac{S_{\mathrm{CO}_{2}}}{K_{\mathrm{CO}_{2}}+S_{\mathrm{CO}_{2}}}
$$

with $I_{\text {out }}(X)=I_{\text {in }} e^{-\left(k X+K_{b g}\right) L}$. 
Based on $[5,14,13]$, the microalgae growth is represented by the following system of differential equations:

$$
\begin{aligned}
\frac{d X}{d t}= & \mu(\cdot) X-D X \\
\frac{d S_{T I C}}{d t}= & -\frac{1}{Y_{C O_{2}}} \mu(\cdot) X+D\left(S_{T I C, i n}-S_{T I C}\right) \\
& +\left(K_{L} a\right)_{C O_{2}}^{P}\left(S_{C O_{2}}^{*}-S_{C O_{2}}\right)+\frac{F}{V}\left(S_{T I C}^{0}-S_{T I C}\right), \\
\frac{d Z}{d t}= & D\left(Z_{i n}-Z\right) .
\end{aligned}
$$

Here, $S_{T I C}$ denotes the concentration of TIC in the PBR, $S_{T I C, \text { in }}$ is the input nutrient concentration (into the $\mathrm{PBR}$ ), and $D>0$ is the dilution rate. The quantity $S_{\mathrm{CO}_{2}}^{*}$ is the concentration of saturation in dissolved $\mathrm{CO}_{2}$ and $\left(K_{L} a\right)_{\mathrm{CO}_{2}}^{P}$ is a transfer coefficient. The term $\left(\mathrm{K}_{\mathrm{L}} \mathrm{a}\right)_{\mathrm{CO}_{2}}^{P}\left(S_{\mathrm{CO}_{2}}^{*}-S_{\mathrm{CO}_{2}}\right)$ represents a liquid-gas transfer between the PBR and the atmosphere. $S_{T I C}^{0}$ represents the TIC concentration in the liquid at the bottom of the column given by the solutions of the BVP $(2.16)-(2.18) \cdot \frac{F}{V}\left(S_{T I C}^{0}-\right.$ $\left.S_{T I C}\right)$ is a circulation term and it represents the TIC exchange with the AC.

To find the oxygen concentration evolution, we solve the following differential equation coupled to (4.5):

$$
\frac{d S_{O_{2}}}{d t}=\frac{1}{Y_{O_{2}}} \mu(\cdot) X+D\left(S_{O_{2}, i n}-S_{O_{2}}\right)+\left(K_{L} a\right)_{O_{2}}^{P}\left(S_{O_{2}}^{*}-S_{O_{2}}\right)+\frac{F}{V}\left(S_{O_{2}}^{0}-S_{O_{2}}\right) .
$$

Here, $S_{\mathrm{O}_{2}}$ denotes the concentration of oxygen in the PBR and $S_{\mathrm{O}_{2} \text {,in }}$ is an input concentration of oxygen (into the PBR). The quantity $S_{\mathrm{O}_{2}}^{*}$ is the concentration of saturation in dissolved $O_{2}$ and $\left(K_{L} a\right)_{O_{2}}^{P}$ is a transfer coefficient. The term $\left(K_{L} a\right)_{O_{2}}^{P}\left(S_{O_{2}}^{*}-S_{O_{2}}\right)$ represents a liquid-gas transfer between the PBR and the atmosphere. $S_{\mathrm{O}_{2}}^{0}$ represents the concentration of $\mathrm{O}_{2}$ in the liquid at the bottom of the column given by the solutions of BVP (2.17)-(2.20). $\frac{F}{V}\left(S_{O_{2}}^{0}-S_{O_{2}}\right)$ is a circulation term and represents the $\mathrm{O}_{2}$ exchange with the AC.

We assume that assumptions A 2.2 and A 2.3 are true in the aqueous medium inside the PBR. This implies that $S_{\mathrm{CO}_{2}}$ equals to $\phi\left(S_{\mathrm{TIC}}, Z(t)\right)$ with $\phi$ defined in Proposition 2.9. In the following section, we discuss the long-term behavior of solutions of (3.4)-(3.5). In particular, we prove that (3.4)-(3.5) admits at most one steady state with a positive microalgae concentration. We denote it by $E_{1}$. We give sufficient conditions for the existence of $E_{1}$ and we argue that starting with a positive initial microalgae concentration any solution of (3.4)-(3.5) approaches asymptotically to $E_{1}$.

4. Asymptotic behavior of the coupled system. In this section, we study the dynamics of the system (3.4)-(3.5). From now on, we assume that (3.4)-(3.5) admits a globally unique solution for any nonnegative initial condition and we write

$$
G\left(S_{T I C}, Z\right)=\left(K_{L} a\right)_{C O_{2}}^{P}\left(S_{C O_{2}}^{*}-\phi\left(S_{T I C}, Z\right)\right)+\frac{F}{V}\left(S_{T I C}^{0}-S_{T I C}\right)
$$

and

$$
H\left(S, S_{O_{2}}, Z\right)=\left(K_{L} a\right)_{O_{2}}^{P}\left(S_{O_{2}}^{*}-S_{O_{2}}\right)+\frac{F}{V}\left(S_{O_{2}}^{0}\left(S_{T I C}, S_{O_{2}}, Z\right)-S_{O_{2}}\right)
$$

Copyright $@$ by SIAM. Unauthorized reproduction of this article is prohibited. 
As shown in section 2 , the term $R:=F\left(S_{T I C}^{0}-S_{T I C}\right)$ is strictly decreasing with respect to $S_{T I C}$ (see (2.22)) and bounded by a positive constant (see (2.23)), while $\phi$ is strictly decreasing in $S_{T I C}$ (see Proposition 2.9). Thus, $G$ is strictly decreasing in $S_{T I C}$ and bounded by a positive constant $G_{0}$. As for $G$, we can argue that $H$ is strictly decreasing in $S_{\mathrm{O}_{2}}$. Since $S_{\mathrm{CO}_{2}}=\phi\left(S_{T I C}, Z\right)$, we can write $\mu(\cdot)=\mu\left(X, S_{T I C}, Z\right)$.

From the third equation in (3.4), it follows that

$$
Z(t)=Z(0) \exp (-D t)+Z_{i n}(1-\exp (-D t))
$$

From (4.3), $Z(t)$ approaches asymptotically $Z_{i n}$. Replacing $Z$ in (3.4) by $Z_{i n}$ and dropping the equation for $Z$, we get the following two-dimensional autonomous system:

$$
\begin{aligned}
\frac{d X}{d t}= & \mu\left(X, S_{T I C}, Z_{i n}\right) X-D X, \\
\frac{d S_{T I C}}{d t}= & -\frac{1}{Y_{C O_{2}}} \mu\left(X, S_{T I C}, Z_{i n}\right) X+D\left(S_{T I C, i n}-S_{T I C}\right) \\
& +G\left(S_{T I C}, Z_{i n}\right) .
\end{aligned}
$$

As a first result, we prove that (4.4) admits a unique steady state characterized by the absence of microalgae.

LEMMA 4.1. (4.4) has a unique washout steady state $\left(0, S_{T I C, 0}\right)$.

Proof. We search for the solution of the following equation:

$$
0=D\left(S_{i n}-S_{T I C}\right)+G\left(S_{T I C}, Z_{i n}\right)=f\left(S_{T I C}\right) .
$$

Since $G$ is strictly decreasing in $S_{T I C}, f$ is strictly decreasing. Thus, (4.5) admits at most one solution. Since $f$ is continuous, $f(0)>0$, and $\lim _{S_{T I C} \rightarrow \infty} f\left(S_{T I C}\right)=-\infty$, the existence of solutions follows.

The following theorem shows that (4.4) admits a globally attracting steady state with a positive microalgae concentration.

TheOREM 4.2. Let $\left(X, S_{T I C}\right)$ be a solution of (4.4) satisfying $S_{T I C}(0) \geq 0$ and $X(0)>0$, and let $S_{T I C, 0}$ be the TIC concentration in absence of microalgae given by Lemma 4.1. Then, if $\mu\left(0, S_{T I C, 0}, Z_{i n}\right)>D$, (4.4) has a unique steady state $\left(X_{1}, S_{T I C, 1}\right)$ with $X_{1}>0$ and it is the limit of $\left(X, S_{T I C}\right)$ as $t \rightarrow \infty$.

Proof. We will simplify the notation by writing $S$ and $\gamma$ instead of $S_{\text {TIC }}$ and $\frac{1}{Y_{\mathrm{CO}_{2}}}$, respectively (and logically $S_{0}$ instead of $\left.S_{T I C, 0}\right)$. Let $(X, S)$ be a solution of (4.4) with initial conditions $S(0) \geq 0$ and $X(0)>0$ and let us define $V:=S+\gamma X$. Since $\mu(X, 0)=0$ and $G\left(0, Z_{i n}\right)>0$, it holds that $X(t), S(t) \geq 0$ for all $t \geq 0$. Then $0 \leq \gamma X(t) \leq Z(t)$ for all $t \geq 0$. Now, we note that $V$ satisfies

$$
\frac{d V(t)}{d t}=D\left(S_{i n}-V\right)+G\left(V(t)-\gamma X(t), Z_{i n}\right) \leq D\left(S_{i n}-V\right)+G\left(0, Z_{i n}\right) .
$$

This shows that $V, X$, and $S$ are bounded. We claim that the limit set of $(X(0), S(0))$ is a single steady state in $\mathbb{R}^{2}$. Indeed, we note that $(V, X)$ is a solution of the system

$$
\begin{aligned}
& \frac{d V}{d t}=D\left(S_{i n}-V\right)+G\left(V-\gamma X, Z_{i n}\right), \\
& \frac{d X}{d t}=\left(\mu\left(X, V-\gamma X, Z_{i n}\right)-D\right) X .
\end{aligned}
$$

Copyright $@$ by SIAM. Unauthorized reproduction of this article is prohibited. 
The Jacobian matrix associated to (4.7) is

$$
J(V, X)=\left[\begin{array}{cc}
\star & -\gamma \frac{\partial}{\partial S} G\left(V-\gamma X, Z_{i n}\right) \\
\frac{\partial}{\partial S} \mu\left(X, V-\gamma X, Z_{i n}\right) X & \star
\end{array}\right] .
$$

It is clear that (4.7) is cooperative (i.e., the off-diagonal terms of the Jacobian matrix are nonnegative). Furthermore, solutions of (4.7) are asymptotically bounded in a compact subset of $\mathbb{R}_{+}^{2}$. From Theorem 2.2 of Chapter 3 in [29] for two-dimensional systems, the limit is a single equilibrium, and the claim is proved.

The Jacobian matrix $J(X, S)$ of the system (4.4) is

$$
\left[\begin{array}{cc}
\frac{\partial \mu\left(X, S, Z_{i n}\right)}{\partial X} X+\mu\left(X, S, Z_{i n}\right)-D & \frac{\partial \mu\left(X, S, Z_{i n}\right)}{\partial S} X \\
-\gamma\left(\frac{\partial \mu\left(X, S, Z_{i n}\right)}{\partial X} X+\mu\left(X, S, Z_{i n}\right)\right) & -\gamma \frac{\partial \mu\left(X, S, Z_{i n}\right)}{\partial S} X-D+\frac{\partial}{\partial S} G\left(S, Z_{i n}\right)
\end{array}\right]
$$

At the washout steady state, the Jacobian matrix is

$$
J\left(0, S_{0}\right)=\left[\begin{array}{cc}
\mu\left(0, S_{0}, Z_{i n}\right)-D & 0 \\
-\gamma \mu\left(0, S_{0}, Z_{i n}\right) & -D+\frac{\partial}{\partial S} G\left(S_{0}, Z_{i n}\right)
\end{array}\right] .
$$

The eigenvalues are $-D+\frac{\partial}{\partial S} G\left(S_{0}, Z_{i n}\right)<0$ and $\mu\left(0, S_{0}, Z_{i n}\right)-D>0$, hence the washout is a saddle point which can be reached only if $X(0)=0$. Consequently, $(X, S)$ cannot converge toward it, and then there is another steady state $\left(X_{1}, S_{1}\right)$ with $X_{1}>0$ the limit of $(X, S)$. This is the unique steady state in $\mathbb{R}_{+}^{2}$ different from the washout. Indeed, assume that there exists another steady state $\left(X_{2}, S_{2}\right)$ with $X_{2}>0$. We can assume that $S_{1}<S_{2}$. Since $\mu\left(X_{1}, S_{1}, Z_{i n}\right)=\mu\left(X_{2}, S_{2}, Z_{\text {in }}\right)$, we have $X_{1}<X_{2}$. Consequently

$$
\varphi\left(S_{1}\right)=\gamma D X_{1}<\gamma D X_{2}=\varphi\left(S_{2}\right),
$$

and we get the contradiction $S_{1}>S_{2}$. Thus, any solution $(X, S)$ to (4.4) with $X(0)>0$ approaches $\left(X_{1}, S_{1}\right)$ and the proof is completed.

The following theorem is an extension of Theorem 4.2.

TheOREM 4.3. Let $\left(X, S_{T I C}, Z\right)$ be a solution of (3.4) satisfying $S(0), Z(0) \geq 0$, and $X(0)>0$, and let $S_{T I C, 0}$ be the TIC concentration in absence of microalgae given by Lemma 4.1. If $\mu\left(0, S_{T I C, 0}, Z_{i n}\right)>D$, then $\left(X, S_{T I C}, Z\right)$ converges to $\left(X_{1}, S_{T I C, 1}, Z_{i n}\right)$, with $\left(X_{1}, S_{T I C, 1}\right)$ given by Theorem 4.2 , as $t \rightarrow \infty$.

Proof. We recall (3.4) with the notation used in the proof of Theorem 4.2. By defining the variable $V=\gamma X+S$ we can prove that the solutions of (3.4) are bounded (see the proof of Theorem 4.2). Let $Y:=\mathbb{R}_{+}^{3}$ and let $\Phi: \mathbb{R}_{+} \times Y \longrightarrow Y$ be the autonomous semiflow defined by $\Phi\left(t, y^{0}\right)=y(t)$ with $y(t)=(X(t), S(t), Z(t))$ the unique solution of (3.4) satisfying $y(0)=y^{0}:=\left(X^{0}, S^{0}, Z^{0}\right) \in Y$. Let $\omega\left(y^{0}\right)$ be the omega limit set of $y^{0}$. The proof of the theorem consists in proving that if $y^{0} \in(0, \infty) \times \mathbb{R}_{+}^{2}$, then $\omega\left(y^{0}\right)=\left\{E_{1}\right\}$ with $E_{1}:=\left(X_{1}, S_{1}, Z_{\text {in }}\right)$.

Since $Z(t)$ converges to $Z_{\text {in }}$ as $t \rightarrow \infty, \omega\left(y^{0}\right) \subset \Gamma:=\mathbb{R}_{+}^{2} \times\left\{Z_{\text {in }}\right\}$. The set $\Gamma$ is invariant and, from Theorem 4.2 , any solution starting on $\Gamma$ approaches either $E_{0}:=$ 
$\left(0, S_{0}, Z_{\text {in }}\right)$ or $E_{1}$. Thus, $\omega\left(y^{0}\right)$ contains $E_{0}$ or $E_{1}$. The characteristic polynomial of the Jacobian evaluated at $E_{1}$ is $p(\lambda)=-(\lambda+D) \operatorname{det}(A-\lambda I)$ with $I$ the identity matrix of $2 \times 2$ and $A$ given by

$$
A=\left[\begin{array}{cc}
X_{1} \frac{\partial}{\partial X} \mu\left(X_{1}, S_{1}, Z_{i n}\right) & X_{1} \frac{\partial}{\partial S} \mu\left(X_{1}, S_{1}, Z_{i n}\right) \\
-\gamma \mu\left(X_{1}, S_{1}, Z_{i n}\right) & -\gamma \frac{\partial}{\partial S} \mu\left(X_{1}, S_{1}, Z_{i n}\right)-D-\frac{\partial}{\partial S} G\left(S_{1}, Z_{i n}\right)
\end{array}\right]
$$

We can easily verify that the trace and the determinant of $A$ are negative and positive, respectively. Thus, all the roots of $p$ are negative. Consequently, $E_{1}$ is locally stable. Thus, if $\omega\left(y^{0}\right)$ contains $E_{1}$, then $\omega\left(y^{0}\right)=\left\{E_{1}\right\}$. Thus, we have to prove that

$$
E_{0} \notin \omega\left(y^{0}\right) \text { for any } y^{0} \in(0, \infty) \times \mathbb{R}_{+}^{2} .
$$

To prove this, we use the theory of persistence presented in the book [30] (we refer the reader to [30] for review of the definitions presented in the rest of the proof). We introduce the persistence function $\rho: Y \longrightarrow \mathbb{R}_{+} ; \rho(y)=X$. From the uniqueness of solutions of (3.4), it follows that

$$
\rho\left(\Phi\left(t, y_{0}\right)\right)=0 \text { for all } t \in \mathbb{R}_{+} \text {if and only if } X^{0}=0 .
$$

Then, we define

$$
Y^{0}:=\left\{y^{0} \in Y ; \rho\left(\Phi\left(t, y^{0}\right)\right)=0\right\}=\{0\} \times \mathbb{R}_{+}^{2} .
$$

From (4.9), we also conclude that $\Phi\left(t,\left(0, S^{0}, Z^{0}\right)\right)=(0, S(t), Z(t))$ with $(S, Z)$ the unique solution of

$$
\begin{aligned}
& \frac{d S}{d t}=D\left(S_{i n}-S\right)+G(S, Z), \\
& \frac{d Z}{d t}=D\left(Z_{i n}-Z\right),
\end{aligned}
$$

satisfying $S(0)=S^{0}$ and $Z(0)=Z^{0}$. It is not difficult to see that any solution of (4.10) approaches asymptotically to the steady state $\left(S_{0}, Z_{i n}\right)$. Therefore, we have that

$$
\Omega:=\bigcup_{y^{0} \in Y^{0}} \omega\left(y^{0}\right)=\bigcup_{S^{0}, Z^{0} \in \mathbb{R}_{+}} \omega\left(0, S^{0}, Z^{0}\right)=\left\{\left(0, S_{0}, Z_{\text {in }}\right)\right\} .
$$

It is clear that $\Omega$ is compact, invariant, isolated, and acyclic in $Y^{0}$.

According to Theorem 8.17 [30], if $\Omega$ is weakly $\rho$-repelling (i.e., there is no $y \in Y$ such that $\rho(y)>0$ and $\Phi(t, y) \rightarrow \Omega$ as $t \rightarrow \infty)$, then $\Phi$ is uniformly weakly $\rho$-persistent (i.e., there exists $\delta>0$ such that $\limsup _{t \rightarrow \infty} \rho(\Phi(t, y))>\delta$ if $\left.\rho(y)>0\right)$. To prove that $\Omega$ is weakly $\rho$-repelling, it is enough to show that $W^{s}(\Omega) \subset Y^{0}$, with $W^{s}(\Omega)$ the stable manifold of $\Omega$. The Jacobian matrix of (3.4) evaluated at the washout steady state is

$$
J=\left[\begin{array}{ccc}
\lambda_{1} & 0 & 0 \\
-\gamma \mu\left(0, S_{0}, Z_{i n}\right) & \lambda_{2} & \frac{\partial}{\partial Z} G\left(S_{0}, Z_{i n}\right) \\
0 & 0 & \lambda_{3}
\end{array}\right]
$$

with $\lambda_{1}=\mu\left(0, S_{0}, Z_{i n}\right)-D, \lambda_{2}=-D+\frac{\partial}{\partial S} G\left(S_{0}, Z_{i n}\right)$, and $\lambda_{3}=-D$ the eigenvalues of $J$. We have $\lambda_{1}>0$, and $\lambda_{2}, \lambda_{3}<0$; therefore $W^{s}(\Omega)$ is a locally two-dimensional 
manifold. Since $Y^{0}$ has dimension 2, we conclude that $W^{s}(\Omega) \subset Y^{0}$. Thus, $\Phi$ is uniformly weakly $\rho$-persistent.

Now we define $\sigma=\rho o \Phi: \mathbb{R}_{+} \times Y \longrightarrow \mathbb{R}_{+}$. It is clear that $\sigma\left(t, y^{0}\right)=X(t)$. It follows that $\sigma$ is continuous, and from the uniqueness of solutions for (3.4), it holds that if $X^{0}>0$, then $\sigma\left(t,\left(X^{0}, S^{0}, Z^{0}\right)\right)>0$ for all $t \geq 0$. The assumptions of Theorem 4.5 in [30] are satisfied, and we conclude that $\Phi$ is uniformly $\rho$-persistent, that is, there exists $\delta>0$ such that

$$
\liminf _{t \rightarrow \infty} \rho\left(\Phi\left(t, y^{0}\right)\right) \geq \delta, \text { for all } y^{0} \in Y ; \rho\left(y^{0}\right)>0,
$$

which is equivalent to

$$
\liminf _{t \rightarrow \infty} X(t) \geq \delta, \text { if } X(0)>0 .
$$

Since (4.11) implies (4.8), the proof is completed.

We end this section studying (3.5). Replacing the state variables $\left(X, S_{T I C}, Z\right)$ by $\left(X_{1}, S_{T I C, 1}, Z_{i n}\right)$ in (3.5), we obtain the one-dimensional equation

$$
\frac{d S_{O_{2}}}{d t}=f\left(S_{O_{2}}\right)
$$

with

$$
f\left(S_{O_{2}}\right)=\frac{1}{Y_{O_{2}}} \mu\left(X_{1}, S_{T I C, 1}, Z_{i n}\right) X_{1}+D\left(S_{O_{2}, i n}-S_{O_{2}}\right)+H\left(S_{T I C, 1}, S_{O_{2}}, Z_{i n}\right) .
$$

Since $f$ is strictly decreasing and continuous, $f(0)>0$, and $\lim _{S \rightarrow \infty} f(S)=-\infty$, (4.12) has a unique steady state $S_{O_{2}, 1}$.

TheOrem 4.4. Let $\left(X, S_{T I C}, Z, S_{\mathrm{O}_{2}}\right)$ be a solution of (3.4)-(3.5) with initial conditions $S_{T I C}(0), Z(0), S_{O_{2}}(0) \geq 0$, and $X(0)>0$, and let $S_{T I C, 0}$ be the TIC concentration without microalgae given by Lemma 4.1. If $\mu\left(0, S_{T I C, 0}, Z_{i n}\right)>D$, then $\left(X, S_{T I C}, Z, S_{O_{2}}\right)$ converges toward $E_{1}:=\left(X_{1}, S_{T I C, 1}, Z_{i n}, S_{O_{2}, 1}\right)$, with $\left(X_{1}, S_{T I C, 1}\right)$ given by Theorem 4.2 , as $t \rightarrow \infty$.

Proof. Let $\left(S, X, Z, S_{O_{2}}\right)$ be a solution of (3.4)-(3.5) with $X(0)>0$. Theorem 4.2 shows that $(X, S, Z)$ approaches asymptotically to the steady state $\left(X_{1}, S_{T I C, 1}, Z_{\text {in }}\right)$. Thus, the omega limit set of this solution is contained in the set $\Omega:=\left\{X_{1}\right\} \times\left\{S_{1}\right\} \times$ $\left\{Z_{\text {in }}\right\} \times \mathbb{R}_{+} . \Omega$ is a positively invariant set with respect to (3.4)-(3.5) and any solution of (3.4)-(3.5) starting in $\Omega$ approaches to $E_{1}$. Thus, $E_{1}$ belongs to the omega limit set of $\left(S, X, Z, S_{\mathrm{O}_{2}}\right)$. If $E_{1}$ is locally stable, then the omega limit set of $\left(S, X, Z, S_{\mathrm{O}_{2}}\right)$ is equal to $\left\{E_{1}\right\}$ and the proof is completed. We omit the proof of the local stability of $E_{1}$ since it is very similar of that of the local stability of $E_{1}$ in the proof of Theorem 4.3.

5. Application to capacity of the coupled system for biogas upgrading. We evaluate numerically the capacity of the system AC-PBR for upgrading biogas at the steady state $E_{1}$. The geometry of the system and flow rates are taken from [20]. Column dimensions are $2.2 \mathrm{~m}$ height and $0.02 \mathrm{~m}$ diameter $\left(S=3.1416 \times 10^{-4} \mathrm{~m}^{2}\right)$. The biogas is injected at a rate of $G=7.9 \mathrm{Ld}^{-1}$. Note that $G_{s}$ in $(2.6)$ is obtained from $G_{S}=x_{C H_{4}} G \rho_{G} / S$, with $x_{C H_{4}}$ the molar fraction of $C H_{4}$ in the gas phase $(0.72)$ and $\rho_{G}$ the molar density of the gas. We will assume that $\rho_{G}=0.0446 \mathrm{~mol} \mathrm{~L}^{-1}$ (density of an ideal gas at $25^{\circ} \mathrm{C}$ and $1 \mathrm{~atm}$ ). The pressure inside the column is taken 

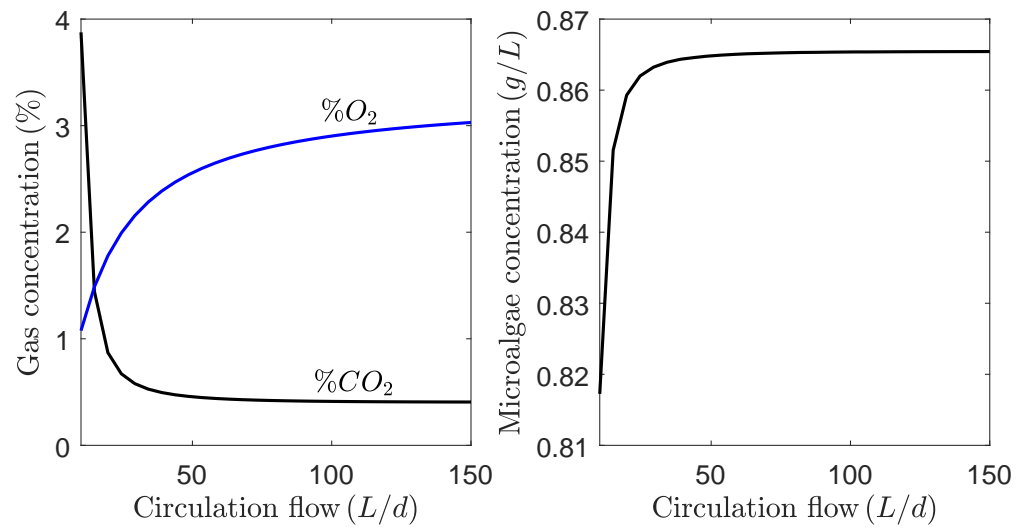

FIG. 3. Biogas composition after passing through the column and microalgae concentration at different steady states reached for different flow liquid rates.

as $P_{0}=1.1064 \mathrm{~atm}$ (average). The PBR has a volume of $75 \mathrm{~L}$ and a depth of $0.15 \mathrm{~m}$. The dilution rate is $0.06 d^{-1}$ and the incident light is $I_{\text {in }}=100 \mu \mathrm{mol} \mathrm{m}^{-2} \mathrm{~s}^{-1}$.

The feed medium has $p H 7$ and concentrations $S_{O_{2}, \text { in }}=3 \times 10^{-4} \mathrm{~mol} \mathrm{~L}^{-1}$ and $S_{T I C, \text { in }}=1.7 \times 10^{-3} \mathrm{~mol} \mathrm{~L}^{-1}$. Thus, $Z_{\text {in }}=1.4 \times 10^{-3} \mathrm{~mol} L^{-1}$. Microalgae parameters are obtained from literature and summarized in Table 2. Gas-liquid transfer coefficients in the column are assumed to be correlated by $\left(k_{L} a\right)_{C_{2}}=\left(k_{L} a\right)_{O_{2}} \sqrt{\frac{D_{C O_{2}}}{D_{O_{2}}}}$, with $\mathrm{D}_{\mathrm{CO}_{2}}$ and $\mathrm{D}_{\mathrm{O}_{2}}$ the diffusivities of $\mathrm{CO}_{2}$ and $\mathrm{O}_{2}$, respectively [16]. The value of $\left(k_{L} a\right)_{O_{2}}$ is $45 d^{-1}$.

Figure 3 shows the composition of the biogas and the microalgae concentration at steady state for different circulation flow rates. The effect of liquid speed $(<0.03 \mathrm{~m} / \mathrm{s})$ on $\left(k_{L} a\right)_{O_{2}}$ is insignificant for industrial applications [27]. Steady state is obtained by integrating (3.4)-(3.5) for 100 days with the solver ode23t of MATLAB. In each iteration, the BVP associated to the AC is solved by bvp4c in MATLAB [28].

By [20], the liquid flow rate is a critical parameter. As the liquid flow rate increases, the percentage of $\mathrm{CO}_{2}$ in treated biogas decreases while the percentage of $\mathrm{O}_{2}$ increases. This suggest that to keep both concentrations below a certain level, intermediate values of the liquid flow rate must be chosen. With respect to the biomass concentration, as in [20], it remains low and almost the same for all the values of the liquid flow rate.

6. Conclusion. We built a mathematical model of an innovative system for purifying biogas: an AC connected to a microalgae culture. The model consists of a system of ordinary differential equations coupled to a BVP. We proved the existence and uniqueness of solutions of the BVP and we found some properties of the solutions. For the coupled system, we determined sufficient conditions for the existence and global attractivity of a steady state characterized by the presence of microalgae.

Based on our theoretical results, we determined numerically the steady states of the coupled system. We showed that the circulation flow between the AC and the reactor with microalgae has a strong impact on the capacity of the system for purifying biogas. 


\section{Appendix A.}

Consider the following problem:

$$
\left\{\begin{aligned}
\frac{d y}{d z} & =G(z, y, y(0)), \\
y(h) & =y_{1}
\end{aligned}\right.
$$

The following lemma gives conditions over $G$ such that the problem (A.1) admits a unique solution.

Lemma A.1. Assume that $G:[0, h] \times \mathbb{R}^{2} \longrightarrow \mathbb{R}$ has the following properties:

(a) the initial value problem (IVP)

$$
\left\{\begin{aligned}
\frac{d y}{d z} & =G(z, y, \gamma), \\
y(0) & =y_{0},
\end{aligned}\right.
$$

admits a unique solution for any $\gamma, y_{0} \in \mathbb{R}$, which exists for all $z \in[0, h]$,

(b) $G$ is Lipschitz in $\gamma$,

(c) there exists $\gamma^{*} \in \mathbb{R}$ such that $G\left(z, \gamma^{*}, \gamma^{*}\right)=0$ for all $z \in[0, h]$,

(d) $G$ is strictly increasing in $\gamma$.

Then, (A.1) admits a unique solution. Moreover, if $\bar{y}\left(z ; y_{1}\right)$ is the solution of (A.1) satisfying $\bar{y}\left(h ; y_{1}\right)=y_{1}$, then $\bar{y}\left(z ; \gamma^{*}\right)=\gamma^{*}$, and $\bar{y}\left(z ; y_{1}\right) \in\left[\gamma^{*}, y_{1}\right]$ (resp., $\bar{y}\left(z ; y_{1}\right) \in$ $\left[y_{1}, \gamma^{*}\right]$ ) for all $z \in[0, h]$ if $y_{1}>\gamma^{*}$ (resp., if $y_{1}<\gamma^{*}$ ).

In the case that $G$ does not depend on $z$, then $\bar{y}\left(z ; y_{1}\right)$ is monotone: increasing if $y_{1}>\gamma^{*}$ and decreasing if $y_{1}<\gamma^{*}$.

Proof. For any $\gamma \in \mathbb{R}$, we denote by $\theta(z ; \gamma)$ the unique solution of (A.2) with $y_{0}=\gamma$.

Claim 1. For any $z \in[0, h], \theta(z ; \cdot)(\theta$ as a function of $\gamma)$ is continuous and strictly increasing on $\mathbb{R}$. Indeed, let $\gamma_{1}, \gamma_{2} \in \mathbb{R}$. Since $G$ is Lipschitz in $\gamma$, we have

$$
\begin{aligned}
\left|\theta\left(z ; \gamma_{1}\right)-\theta\left(z ; \gamma_{2}\right)\right| & \leq\left|\gamma_{1}-\gamma_{2}\right|+\int_{0}^{z}\left|G\left(z, y, \gamma_{1}\right)-G\left(z, y, \gamma_{2}\right)\right|, \\
& \leq\left(1+L_{G} h\right)\left|\gamma_{1}-\gamma_{2}\right|
\end{aligned}
$$

with $L_{G}$ the Lipschitz constant of $G$. Then $\theta(z ; \cdot)$ is Lipschitz in $\gamma$ and hence continuous in $\gamma$. For the second result, we will denote by $\theta\left(z ; \gamma, \gamma^{\prime}\right)$ the unique solution of the differential equation $d y / d z=G(z, y, \gamma)$ with initial condition $y(0)=\gamma^{\prime}$, which exists for all $z \in[0, h]$. Assume that $\gamma_{1}<\gamma_{2}$; then $G\left(z, y, \gamma_{1}\right)<G\left(z, y, \gamma_{2}\right)$ for all $y \in \mathbb{R}$. By the standard comparison theorem, it follows that $\theta\left(z ; \gamma_{1}, \gamma_{1}\right)<\theta\left(z ; \gamma_{1}, \gamma_{2}\right)$. From a uniqueness argument, it follows that $\theta\left(z ; \gamma_{1}, \gamma_{2}\right)<\theta\left(z ; \gamma_{2}, \gamma_{2}\right)$. Combining the last two inequalities for $\theta$, we conclude that $\theta\left(z ; \gamma_{1}\right)<\theta\left(z ; \gamma_{2}\right)$, and hence $\theta(z ; \gamma)$ is strictly increasing in $\gamma$.

Claim 2. We have that $\theta\left(z, \gamma^{*}\right)=\gamma^{*}$ and that $\theta(z, \gamma)>\gamma($ resp., $\theta(z, \gamma)>\gamma)$ for all $z \in[0, h]$ when $\gamma>\gamma^{*}$ (resp., $\gamma<\gamma^{*}$ ). Indeed, from (c) we have that $G\left(z, \gamma^{*}, \gamma^{*}\right)=0$ for all $z \in[0, h]$, from where it follows that $\theta\left(z, \gamma^{*}\right)$ is constant and equals to $\gamma^{*}$. Let $\gamma>\gamma^{*}$ (resp., $\gamma<\gamma^{*}$ ). Since $G$ is strictly increasing in $\gamma$, for any $z \in[0, h]$ we have

$$
\left.\left.\frac{d \theta(z, \gamma)}{d z}\right|_{\theta=\gamma^{*}}=G\left(z, \gamma^{*}, \gamma\right)>G\left(z, \gamma^{*}, \gamma^{*}\right)=0 \text { (resp., }\left.\frac{d \theta(z, \gamma)}{d z}\right|_{\theta=\gamma^{*}}<0\right) .
$$

Thus, the region $\left[\gamma^{*}, \infty\right)$ (resp., $\left.\left(-\infty, \gamma^{*}\right]\right)$ is positively invariant and the claim is proved. 
Now, define the function $\varphi: \mathbb{R} \longrightarrow \mathbb{R}$ as $\varphi(\gamma):=\theta(h, \gamma)$. It remains to prove that there is a unique $\gamma^{\prime} \in \mathbb{R}$ such that $\varphi\left(\gamma^{\prime}\right)=y_{1}$. Indeed, from Claim 2, $\varphi\left(\gamma^{*}\right)=\gamma^{*}$, $\varphi(\gamma)>\gamma$ when $\gamma>\gamma^{*}$ and $\varphi(\gamma)<\gamma$ when $\gamma<\gamma^{*}$. This implies that $\varphi$ is surjective. From Claim 1, it follows that $\varphi$ is continuous and strictly increasing. Thus, $\varphi$ is a homeomorphism and we conclude the existence and uniqueness of $\gamma^{\prime}$.

If $G$ does not depend on $z$, (A.3) implies (in a first order autonomous differential equation) that $\theta(z, \gamma)$ is a strictly increasing (resp., strictly decreasing) function for all $z \in[0, h]$. This completes the proof.

Lemma A.2. Assume that for any $z \in[0, h], G(z, \cdot) \in C^{1}\left(\mathbb{R}^{2}\right)$ and

$$
\frac{\partial}{\partial y} G(z, y, \gamma)+\frac{\partial}{\partial \gamma} G(z, y, \gamma)>0
$$

Assume also that for any $y_{1} \in \mathbb{R}$ (A.1) admits a unique solution $\bar{y}\left(z ; y_{1}\right)$. Then, $\bar{y}\left(0 ; y_{1}\right)$ is differentiable by $y_{1}$ and

$$
0 \leq \frac{\partial \bar{y}\left(0 ; y_{1}\right)}{\partial y_{1}} \leq 1
$$

Proof. For any $\gamma \in \mathbb{R}$, we will denote by $\theta(z ; \gamma)$ the unique solution of the IVP (A.2) with $y_{0}=\gamma$. Following the proofs of Theorems 12.2 and 12.4 in [2], it can be shown that $w(z):=\frac{\partial}{\partial \gamma} \theta(z, \gamma)$ exists for all $z \in[0, h]$ and it satisfies the variational equation

$$
\frac{d w(z)}{d z}=\frac{\partial}{\partial y} G(z, \theta(z, \gamma), \gamma) w(z)+\frac{\partial}{\partial \gamma} G(z, \theta(z, \gamma), \gamma)=F(z, w(z))
$$

with the initial condition $w(0)=1$. We have $F(z, 1)>0$ and hence $w(z)$ is greater than 1 for all $z \in(0, h]$. Now, let $\bar{y}$ be a solution of the problem (A.1). We have that $\theta(h, \bar{y}(0))=y_{1}$. By taking the derivative with respect to $y_{1}$ on both sides of this expression we get

$$
\frac{\partial \theta(h, \bar{y}(0))}{\partial \bar{y}(0)} \frac{\partial \bar{y}(0)}{\partial y_{1}}=1
$$

The proof follows from the fact that $w(h)=\frac{\partial \theta(h, \bar{y}(0))}{\partial \bar{y}(0)} \geq 1$.

\section{REFERENCES}

[1] N. Abatzoglou and S. Boivin, A review of biogas purification processes, Biofuels Bioproducts Biorefining, 3 (2009), pp. 42-71.

[2] R. P. Agarwal and D. O'Regan, An Introduction to Ordinary Differential Equations, Springer, New York, 2008.

[3] P. Amster, Topological Methods in the Study of Boundary Value Problems, Springer, New York, 2014.

[4] Z. BAI AND W. GE, Existence of three positive solutions for some second-order boundary value problems, Comput. Math. Appl., 48 (2004), pp. 699-707.

[5] O. Bernard, Hurdles and challenges for modelling and control of microalgae for CO2 mitigation and biofuel production, J. Process Control, 60 (2011), pp. 1378-1389.

[6] J. Conde, L. Moro, L. Travieso, E. Sanchez, A. Leiva, R. Dupeiron, and R. EsCOBEDO, Biogas purification process using intensive microalgae cultures, Biotechnol. Lett., 15 (1993), pp. 317-320.

[7] A. Converti, R. Oliveira, B. Torres, A. Lodi, and M. Zilli, Biogas production and valorization by means of a two-step biological process, Bioresource Technol., 100 (2009), pp. 5771-5776.

Copyright $@$ by SIAM. Unauthorized reproduction of this article is prohibited. 
[8] W. A. Coppel, Stability and Asymptotic Behavior of Differential Equations, Heath, Lexington, MA, 1965.

[9] B. Decostere, N. Janssens, A. Alvarado, T. Maere, P. Goethals, S. W. Van Hulle, AND I. Nopens, A combined respirometer-titrimeter for the determination of microalgae kinetics: Experimental data collection and modelling, Chemical Eng. J., 222 (2013), pp. 85-93.

[10] W. K. Dodds, Freshwater Ecology: Concepts and Environmental Applications, Elsevier, Amsterdam, 2002.

[11] H. S. HARned AND R. DAVIS, JR., The ionization constant of carbonic acid in water and the solubility of carbon dioxide in water and aqueous salt solutions from 0 to 50, J. Amer. Chemical Soc., 65 (1943), pp. 2030-2037.

[12] C.-H. Hsien AND W.-T. WU, A novel photobioreactor with transparent rectangular chambers for cultivation of microalgae, Biochemical Eng. J., 46 (2009), pp. 300-305.

[13] J. Huisman, H. C. Matthijs, P. M. Visser, H. Balke, C. A. Sigon, J. Passarge, F. J. Weissing, And L. R. Mur, Principles of the light-limited chemostat: Theory and ecological applications, Antonie van Leeuwenhoek, 81 (2002), pp. 117-133.

[14] G. A. Ifrim, M. Titica, G. Cogne, L. Boillereaux, J. Legrand, and S. Caraman, Dynamic ph model for autotrophic growth of microalgae in photobioreactor: A tool for monitoring and control purposes, AIChE J., 60 (2014), pp. 585-599.

[15] N. Kantarci, F. Borak, and K. O. Ulgen, Bubble column reactors, Process Biochem., 40 (2005), pp. 2263-2283.

[16] C. G. Khoo, M. K. LAm, AND K. T. Lee, Pilot-scale semi-continuous cultivation of microalgae chlorella vulgaris in bubble column photobioreactor (BC-PBR): Hydrodynamics and gasliquid mass transfer study, Algal Res., 15 (2016), pp. 65-76.

[17] W. Lewis and W. Whitman, Principles of gas absorption, Industrial Eng. Chemistry, 16 (1924), pp. 1215-1220.

[18] G. Mandeno, R. Craggs, C. Tanner, J. Sukias, and J. Webster-Brown, Potential biogas scrubbing using a high rate pond, Water Sci. Technol., 51 (2005), pp. 253-256.

[19] J. Mata-Alvarez, S. Mace, And P. Llabres, Anaerobic digestion of organic solid wastes. an overview of research achievements and perspectives, Bioresource Technol., 74 (2000), pp. $3-16$.

[20] L. Meier, R. Pérez, L. Azócar, M. Rivas, and D. Jeison, Photosynthetic CO2 uptake by microalgae: An attractive tool for biogas upgrading, Biomass Bioenergy, 73 (2015), pp. $102-109$.

[21] R. W. Missen, C. A. Mims, And B. A. Saville, Introduction to Chemical Reaction Engineering and Kinetics, Wiley, New York, 1999.

[22] R. Muñoz, L. Meier, I. Diaz, and D. Jeison, A review on the state-of-the-art of physical/chemical and biological technologies for biogas upgrading, Rev. Environmental Sci. Bio/Technology, 14 (2015), pp. 727-759.

[23] J. T. Novak AND D. E. BRune, Inorganic carbon limited growth kinetics of some freshwater algae, Water Research, 19 (1985), pp. 215-225.

[24] W. Rudin, Principles of Mathematical Analysis, Internat. Ser. Pure Appl. Math., McGraw-Hill, New York, 1976.

[25] R. SAnder, Compilation of Henry's law constants (version 4.0) for water as solvent, Atmos. Chem. Phys., 15 (2015).

[26] M. L. Serejo, E. Posadas, M. A. Boncz, S. Blanco, P. García-Encina, and R. Munoz, Influence of biogas flow rate on biomass composition during the optimization of biogas upgrading in microalgal-bacterial processes, Environ. Sci. Technol., 49 (2015), pp. 3228-3236.

[27] Y. Shah, B. G. Kelkar, S. Godbole, and W.-D. Deckwer, Design parameters estimations for bubble column reactors, AIChE J., 28 (1982), pp. 353-379.

[28] L. F. Shampine, J. Kierzenka, and M. W. Reichelt, Solving Boundary Value Problems for Ordinary Differential Equations in MATLAB with bvp $4 c$, Tutorial notes, MATLAB Central File Exchange, 2000, pp. 1-27.

[29] H. L. Smith, Monotone Dynamical Systems: An Introduction to the Theory of Competitive and Cooperative Systems, Math. Surveys Monogr. 41, AMS, Providence, RI, 2008.

[30] H. L. Smith and H. R. Thieme, Dynamical Systems and Population Persistence, Grad. Stud. Math. 118, AMS, Providence, RI, 2011.

[31] P. Spolaore, C. Joannis-Cassan, E. Duran, and A. Isambert, Commercial applications of microalgae, J. Biosci. Bioengrg., 101 (2006), pp. 87-96.

[32] A. Toledo-Cervantes, C. Madrid-Chirinos, S. Cantera, R. Lebrero, and R. Muñoz, Influence of the gas-liquid flow configuration in the absorption column on photosynthetic biogas upgrading in algal-bacterial photobioreactors, Bioresource Technol., 225 (2017), pp. $336-342$.

Copyright (c) by SIAM. Unauthorized reproduction of this article is prohibited. 
[33] E. I. Volcke, S. Van Hulle, T. Deksissa, U. Zaher, and P. Vanrolleghem, Calculation of $p H$ and Concentration of Equilibrium Components During Dynamic Simulation by Means of a Charge Balance, BIOMATH Technical report, Ghent University, Ghent, Belgium, 2005.

[34] J. R. Welty, C. E. Wicks, G. Rorrer, and R. E. Wilson, Fundamentals of Momentum, Heat, and Mass Transfer, Wiley, New York, 2009.

[35] R. H. Wijffels and M. J. Barbosa, An outlook on microalgal biofuels, Science, 329 (2010), pp. $796-799$.

Copyright (c) by SIAM. Unauthorized reproduction of this article is prohibited. 